\title{
Thermal effects on the catalysis by a magnetic field
}

\author{
Ashok Das and Marcelo Hott* \\ Department of Physics and Astronomy, University of Rochester, Rochester, New York 14627
}

(Received 17 April 1995)

\begin{abstract}
We show that the formation of condensates in the presence of a constant magnetic field in $2+1$ dimensions is extremely unstable. It disappears as soon as a heat bath is introduced with or without a chemical potential. The value of the condensate as well as other observables are shown to become nonanalytic at finite temperature.
\end{abstract}

PACS number(s): 11.10.Kk, 11.10.Wx, 11.30.Qc

\section{INTRODUCTION}

Induced quantum numbers in (2+1)-dimensional quantum field theories have been investigated in detail in the past [1-4]. There has been renewed interest in the subject [5-7] after the suggestion that in the presence of a constant magnetic field, in 2+1 dimensions, flavor symmetry is broken and fermions can generate a mass even at the weakest attractive interaction between the fermions $[5,6]$. More precisely, it was shown in [6] that in the presence of a constant magnetic field, Dirac fermions (with two flavors combined into a four-component spinor) develop a nonzero value for the condensate leading to a breakdown of the flavor symmetry. This, however, does not give the fermions a mass. On the other hand, if one now introduces an interaction of the Nambu-Jona-Lasinio-type, a fermionic mass is shown to be generated for any value of the interaction $[5,6]$, which can be physically thought of as arising due to the nonzero value of the condensate in the presence of the magnetic field.

The calculation of the condensates involves regularizing the infrared modes with a fermion mass term which is taken to zero at the end. In $2+1$ dimensions, the value of the condensate is a discontinuous function of the fermion mass parameter and depends on how the zero-mass limit is taken. (More explicitly, the condensate depends on the sign of the mass term in the Lagrangian.) A nonzero value of the condensate implies the breakdown of the flavor symmetry (chiral symmetry) of the system. In this Brief Report, we will show that this formation of the condensates is a very unstable phenomenon. The condensates disappear as soon as a heat bath is introduced (for any finite temperature) with or without a chemical potential. Furthermore, the theory develops a nonanalytic behavior at the zero-temperature limit. The paper is organized as follows. In Sec. II, we recapitulate

* On leave of absence from UNESP-Campus de Guaratinguetá, P.O. Box 205, CEP:12.500, Guaratinguetá, S.P., Brazil. briefly the calculation of the value of the condensate at zero temperature in a manner that extends to a finitetemperature analysis naturally. In Sec. III, we show that the condensate disappears for any finite value of the temperature. We point out the nonanalytic structure of the condensates in the limit of zero temperature. We discuss the behavior of the thermal Bogoliubov transformations and show that the generator of these transformations becomes nonanalytic in this limit as well. We present a short discussion of this behavior of the condensates in Sec. IV.

\section{ZERO TEMPERATURE}

The calculation of the condensate, in the present theory, is carried out in [6] in a proper time representation which is not very suitable for extension to finite temperature. In this section, therefore, we give an alternate derivation of the zero-temperature result which readily extends to finite temperature. We follow the notation of [6] for simplicity and take the Lagrangian for the theory in $2+1$ dimensions to be

$$
\mathcal{L}=\bar{\Psi}\left[\gamma^{\mu}\left(i \partial_{\mu}-e A_{\mu}\right)-m\right] \Psi
$$

where $\Psi$ is a four-component spinor involving two flavors, $e$ is the electric charge, and we choose the electromagnetic potential to have the form $A_{\mu}=(0,0, B x)$ with $B$ representing the constant, external magnetic field. The Landau levels can be easily calculated [8] and have the energy values (in our entire discussions, we will assume that $e B>0$ )

$$
E_{n}= \pm \sqrt{2 e B n+m^{2}}, \quad n=0,1,2, \ldots
$$

with a double degeneracy for the $n \neq 0$ modes. The degeneracy in the $y$ component of the momentum, $p_{y}=$ $p$ is also understood. The orthonormal positive- and negative-energy eigenstates can also be worked out in a straightforward manner and have the forms 


$$
\begin{aligned}
& \psi_{1}^{(+)}(n, p, \vec{x}, t)= N_{n} \exp \left[-i\left(\left|E_{n}\right| t-p y\right)\right] \\
& \times\left(\begin{array}{c}
\left(\left|E_{n}\right|+m\right) I(n, p, x) \\
-\sqrt{2 e B n} I(n-1, p, x) \\
0 \\
0
\end{array}\right), \\
& \psi_{2}^{(+)}(n, p, \vec{x}, t)= N_{n} \exp \left[-i\left(\left|E_{n}\right| t-p y\right)\right] \\
& 0 \\
& 0 \\
& \times\left(\begin{array}{c}
-\sqrt{2 e B n} I(n, p, x) \\
\left(\left|E_{n}\right|+m\right) I(n-1, p, x)
\end{array}\right),
\end{aligned}
$$

$$
\begin{aligned}
\psi_{1}^{(-)}(n, p, \vec{x}, t)= & N_{n} \exp \left[i\left(\left|E_{n}\right| t-p y\right)\right] \\
& \times\left(\begin{array}{c}
\sqrt{2 e B n} I(n,-p, x) \\
\left(\left|E_{n}\right|+m\right) I(n-1,-p, x) \\
0 \\
0
\end{array}\right),
\end{aligned}
$$

$$
\begin{aligned}
\psi_{2}^{(-)}(n, p, \vec{x}, t)= & N_{n} \exp \left[i\left(\left|E_{n}\right| t-p y\right)\right] \\
& \times\left(\begin{array}{c}
0 \\
0 \\
\left(\left|E_{n}^{-}\right|+m\right) I(n,-p, x) \\
\sqrt{2 e B n} I(n-1,-p, x)
\end{array}\right),
\end{aligned}
$$

where

$$
\begin{gathered}
N_{n}=\frac{1}{\sqrt{2\left|E_{n}\right|\left(\left|E_{n}\right|+m\right)}} \\
I(n, p, x)=\left(\frac{e B}{\pi}\right)^{1 / 4} \frac{1}{\sqrt{2^{n} n !}} \exp \left[-\frac{e B}{2}\left(x-\frac{p}{e B}\right)^{2}\right] \\
\times H_{n}[\sqrt{e B}(x-p / e B)] \\
I(n=-1, p, x)=0
\end{gathered}
$$

with the $H_{n}$ 's representing the Hermite polynomials. There are only two ground-state wave functions as can be seen from Eq. (3). Their structure depends on the sign of the mass term. For example, for $m>0$, the two ground-state wave functions have the simpler form

$$
\begin{aligned}
& \psi^{(+)}(0, p, \vec{x}, t)=\exp \left[-i\left(\left|E_{0}\right| t-p y\right)\right]\left(\begin{array}{c}
I(0, p, x) \\
0 \\
0 \\
0
\end{array}\right), \\
& \psi^{(-)}(0, p, \vec{x}, t)=\exp \left[i\left(\left|E_{0}\right| t-p y\right)\right]\left(\begin{array}{c}
0 \\
0 \\
I(0,-p, x) \\
0
\end{array}\right) .
\end{aligned}
$$

To calculate the condensate, we expand the field operator in the basis of these wave functions as

$$
\begin{aligned}
\Psi(\vec{x}, t)= & \sum_{n} \sum_{i=1,2}{ }^{\prime} \int \frac{d p}{\sqrt{2 \pi}}\left[a_{i}(n, p) \psi_{i}^{(+)}\right. \\
& \left.+b_{i}^{\dagger}(n, p) \psi_{i}^{(-)}\right]
\end{aligned}
$$

where the prime in the sum over $i$ represents the fact that this sum is only for the $n \neq 0$ modes. The creation and the annihilation operators for the particle and the antiparticles satisfy the standard anticommutation relations, e.g.,

$$
\begin{aligned}
{\left[a_{i}(n, p), a_{j}^{\dagger}\left(n^{\prime}, p^{\prime}\right)\right]_{+} } & =\delta_{i j} \delta_{n n^{\prime}} \delta\left(p-p^{\prime}\right) \\
& =\left[b_{i}(n, p), b_{j}^{\dagger}\left(n^{\prime}, p^{\prime}\right)\right]_{+}
\end{aligned}
$$

with all others vanishing. It is now straightforward to evaluate the condensate which has the value

$$
\langle 0|\bar{\Psi}(\vec{x}, t) \Psi(\vec{x}, t)| 0\rangle=-\frac{m}{|m|} \frac{e B}{2 \pi}-\frac{m e B}{\pi} \sum_{n=1}^{\infty} \frac{1}{E_{n}},
$$

where $E_{n}$ stands for the positive root given in Eq. (2). The second sum on the right-hand side is a Hurwitz $\zeta$ function which in the limit of vanishing $m$ reduces to the Riemann $\zeta$ function, $\zeta_{R}(1 / 2)[9]$. The Riemann $\zeta$ function $\zeta_{R}(s)$ is an analytic function [10] in the entire complex plane except for a simple pole at $s=1$. Its value at $s=\frac{1}{2}$ is known to be $\zeta_{R}(1 / 2)=-1.46[10,11]$. Thus, we see that in the limit of vanishing mass, the condensate has the value

$$
\langle 0|\bar{\Psi}(\vec{x}, t) \Psi(\vec{x}, t)| 0\rangle=-\frac{m}{|m|} \frac{e B}{2 \pi} .
$$

This is the same value as [6] (except for the missing sign of the mass factor there).

\section{FINITE TEMPERATURE}

The transition to finite temperature is now straightforward. We use thermofield dynamics [12] for our discussion. Introducing a tilde field, we note that we can write a thermal doublet of fermionic fields as [12-14]

$$
\Phi(\vec{x}, t)=\left(\begin{array}{c}
\Psi(\vec{x}, t) \\
\tilde{\Psi}^{\dagger}(\vec{x}, t)
\end{array}\right),
$$

which can be expanded in terms of the wave functions derived earlier as

$$
\begin{aligned}
\Phi(\vec{x}, t)= & \sum_{n} \sum_{1=1,2} \prime \int \frac{d p}{\sqrt{2 \pi}}\left[\left(\begin{array}{c}
a_{i}(n, p) \\
\tilde{a}_{i}^{\dagger}(n, p)
\end{array}\right) \psi_{i}^{(+)}(n, p, \vec{x}, t)\right. \\
& \left.+\left(\begin{array}{c}
b_{i}^{\dagger}(n, p) \\
\tilde{b}_{i}(n, p)
\end{array}\right) \psi_{i}^{(-)}(n, p, \vec{x}, t)\right] .
\end{aligned}
$$

We can now introduce the thermal Bogoliubov transformation of the form 


$$
U(\theta)=\exp [i G(\theta)],
$$

where the generator of the transformation is given by

$$
\begin{aligned}
G(\theta)= & i \sum_{n} \sum_{i=1,2}{ }^{\prime} \int d p\left[\theta_{i}^{(+)}(n)\left(\tilde{a}_{i} a_{i}-a_{i}^{\dagger} \tilde{a}_{i}^{\dagger}\right)\right. \\
& \left.+\theta_{i}^{(-)}(n)\left(\tilde{b}_{i} b_{i}-b_{i}^{\dagger} \tilde{b}_{i}^{\dagger}\right)\right],
\end{aligned}
$$

and

$$
\begin{gathered}
\sin ^{2} \theta_{i}^{(+)}(n)=n_{F}\left(E_{n}\right), \\
\sin ^{2} \theta_{i}^{(-)}(n)=1-n_{F}\left(-E_{n}\right), \\
n_{F}\left(E_{n}\right)=\frac{1}{\exp \left[\beta\left(E_{n}-\mu\right)\right]+1} .
\end{gathered}
$$

$$
\begin{aligned}
\langle 0, \beta|\Psi \Psi \Psi| 0, \beta\rangle= & \sum_{n} \sum_{i=1,2} \int^{\prime} \frac{d p}{2 \pi}\left[\sin ^{2} \theta_{i}^{(+)}(n) \bar{\psi}_{i}^{(+)} \psi_{i}^{(+)}+\cos ^{2} \theta_{i}^{(-)}(n) \bar{\psi}_{i}^{(-)} \psi_{i}^{(-)}\right] \\
= & \frac{m}{|m|} \frac{e B}{2 \pi}\left(1-\frac{1}{\exp [\beta|m|-\mu]+1}-\frac{1}{\exp [\beta(|m|+\mu)]+1}\right) \\
& -\frac{m e B}{\pi} \sum_{n=1}^{\infty} \frac{1}{E_{n}}\left(1-\frac{1}{\exp \left[\beta\left(E_{n}-\mu\right)\right]+1}-\frac{1}{\exp \left[\beta\left(E_{n}+\mu\right)\right]+1}\right) .
\end{aligned}
$$

It is clear now that, in the limit $m \rightarrow 0$ (for finite $\beta$ ),

$$
\langle 0, \beta|\bar{\Psi} \Psi| 0, \beta\rangle=0 \text {. }
$$

That is, the condensate vanishes for any finite, nonzero temperature.

It is also clear now that the structure of the condensate exhibits a nonanalyticity at finite temperature. This is best seen by setting $\mu=0$. In this case, we note that we can take the limit

$$
m \rightarrow 0, \quad \beta \rightarrow \infty, \quad|m| \beta=\alpha .
$$

Then, the value of the condensate, in this limit, can be obtained from Eq. (17) to be

$$
\langle 0, \beta|\Psi \Psi| 0, \beta\rangle \rightarrow-\frac{m}{|m|} \frac{e B}{2 \pi}\left(1-\frac{2}{\exp (\alpha)+1}\right) .
$$

This shows that the order of the limits $m \rightarrow 0$ and $\beta \rightarrow \infty$ is not commutative. That is, the condensate is not analytic at $T=0$. Finite-temperature field theories are known to exhibit nonanalyticity $[15,16]$ in the energymomentum variables, but this is a nonanalyticity in the value of the condensate at the origin in the $(m, T)$ plane. We would like to point out here that similar nonanalyticity has also been noted earlier in the study of induced quantum numbers at finite temperature [17].

To understand the peculiar behavior of the condensate as well as the nonanalyticity at finite temperature,
Here $\beta=1 / k T$ and $\mu$ represents the chemical potential. The thermal vacuum can now be defined as

$$
|0, \beta\rangle=U(\theta)|0, \tilde{0}\rangle
$$

The thermal creation and annihilation operators, similarly, can be obtained through the Bogoliubov transformation to be

$$
\begin{aligned}
& \left(\begin{array}{c}
a_{i}^{\beta}(n, p) \\
\tilde{a}_{i}^{\beta \dagger}(n, p)
\end{array}\right) \\
& \quad=\left(\begin{array}{cc}
\cos \theta_{i}^{(+)}(n) & -\sin \theta_{i}^{(+)}(n) \\
\sin \theta_{i}^{(+)}(n) & \cos \theta_{i}^{(+)}(n)
\end{array}\right)\left(\begin{array}{c}
a_{i}(n, p) \\
\tilde{a}_{i}^{\dagger}(n, p)
\end{array}\right)
\end{aligned}
$$

and similarly for $b_{i}$ and $\tilde{b}_{i}$.

Given these, we can now calculate the value of the condensate in the thermal vacuum simply as we analyze next the structure of the Bogoliubov transformation in Eqs. (12) and (13). And to simplify the analysis, we set the chemical potential $\mu=0$. The first simplification that occurs in this case is that

$$
\theta_{i}^{(+)}(n)=\theta_{i}^{(-)}(n)
$$

and from Eq. (14), we note that there is really one $\theta$ for every mode satisfying

$$
\tan \theta(n)=\exp \left(-\beta E_{n} / 2\right) .
$$

The generator of the Bogoliubov transformation, in this case, has the form

$$
\begin{aligned}
G(\theta)= & i \sum_{n} \sum_{i=1,2}{ }^{\prime} \int d p \theta(n)\left(\tilde{a}_{i} a_{i}-a_{i}^{\dagger} \tilde{a}_{i}^{\dagger}\right. \\
& \left.+\tilde{b}_{i} b_{i}-b_{i}^{\dagger} \tilde{b}_{i}^{\dagger}\right) .
\end{aligned}
$$

For $m \neq 0$, this indeed has the right behavior in that, as $\beta \rightarrow \infty$,

$$
\tan \theta(n)=0 \Rightarrow \theta(n)=0
$$

so that the Bogoliubov transformation simply reduces to the identity opexator. However, for any finite $\beta$ or temperature, if we let $m \rightarrow 0$, we note from Eq. (21) as well as from the definition of $E_{n}$ that

$$
\tan \theta(n=0)=1 \Rightarrow \theta(n=0)=\pi / 4 \text {. }
$$


It is, of course, the ground state that contributes to the condensate in the vanishing mass limit and we see that for this mode $\theta$ has the unique value of $\pi / 4$. The thermal states generated by the Bogoliubov transformations can be thought of as squeezed states [18] which in some sense are polarized states. More specifically, for a four-mode system, as is the case here, $(a, \tilde{a}, b, \tilde{b})$, it can be easily checked that in a squeezed state parametrized by an angle $\theta$ :

$$
\left\langle\theta\left|a^{\dagger} a-b b^{\dagger}\right| \theta\right\rangle=-\cos 2 \theta .
$$

Consequently, for $\theta=\pi / 4$, the squeezed state acts as a crossed polarizer for the expectation value of this operator which vanishes. (This is the operator expectation value for the lowest mode which is responsible for the value of the condensate and this is how the value of the condensate vanishes.)

Let us also note that in the limit of Eq. (19), all the $\theta(n)$ 's vanish except for the lowest-energy mode which gives

$$
\tan \theta(n=0)=\exp \left(-\frac{\alpha}{2}\right) \Rightarrow \theta(n=0)=\theta(\alpha) .
$$

That is, in this case, the Bogoliubov transformation does not reduce to the identity operator and, in fact, depends on how the zero-temperature and the zero-mass limit is taken. This is the nonanalyticity in the condensates that we discussed earlier and we see that in this case, the generator of the Bogoliubov transformation, itself, becomes nonanalytic. Consequently, not only is the value of the condensate nonanalytic at the origin in the $(m, T)$ plane, but most observables are likely to be.

\section{CONCLUSION}

In this paper, we have pointed out that the formation of condensates in a (2+1)-dimensional field theory, in the presence of a constant, external magnetic field, is a highly unstable phenomenon. As soon as a heat bath is introduced, the value of the condensate vanishes for any finite temperature. The thermal expectation value appears to average over domains with opposite values for the condensate (very much like the double-well example), leading to a vanishing value for the condensate. Furthermore, as we have pointed out, the theory develops a nonanalyticity in this case in that most observables including the value of the condensate appear to be nonanalytic at the origin in the $(m, T)$ plane.

\section{ACKNOWLEDGMENTS}

One of us (A.D.) would like to thank Professor N. P. Chang for discussions. This work was supported in part by the U.S. Department of Energy Grant No. DE-FG-0291ER40685. M.H. would like to thank the Fundação de Amparo a Pesquisa do Estado de São Paulo for financial support.
[1] A. Niemi and G. Semenoff, Phys. Rev. Lett. 51, 2077 (1983).

[2] A. Redlich, Phys. Rev. Lett. 52, 18 (1984); Phys. Rev. D 29, 2366 (1984).

[3] M. B. Paranjape, Phys. Rev. Lett. 55, 2390 (1985).

[4] D. Boyanovsky, R. Blankenbecler, and R. Yahalom, Nucl. Phys. B270, 483 (1986); Phys. Rev. D 34, 612 (1986).

[5] K. G. Klimenko, Z. Phys. C 54, 323 (1992).

[6] V. P. Gusynin, V. A. Miransky, and I. A. Shovkovy, Phys. Rev. Lett. 73, 3499 (1994).

[7] D. Cangemi, E. D'Hoker, and G. V. Dunne, Phys. Rev. D 51, R2513 (1995); R. Parwani, Phys. Lett. B 358, 101 (1995).

[8] M. Kobayashi and M. Sakamoto, Prog. Theor. Phys. 70, 1375 (1983).

[9] See, for example, S. Blau, M. Visser, and A. Wipf, Int. J. Mod. Phys. A 6, 5409 (1991).

[10] H. M. Edwards, Riemann's Zeta Function (Academic,
New York, 1974).

[11] E. Jahnke and F. Emde, Tables of Functions (Dover, New York, 1956).

[12] H. Umezawa, H. Matsumoto, and M. Tachiki, Thermo Field Dynamics (North-Holland, Amsterdam, 1982).

[13] I. Ojima, Ann. Phys. (N.Y.) 137, 1 (1981).

[14] P. Elmfors, D. Persson, and B. S. Skagerstam, Phys. Rev. Lett. 71, 480 (1993); see also Astropart. Phys. 2, 299 (1994).

[15] H. A. Weldon, Phys. Rev. D 26, 1394 (1982); 28, 2007 (1983).

[16] H. A. Weldon, Phys. Rev. D 47, 594 (1993); P. F. Bedaque and A. Das, ibid. 47, 601 (1993).

[17] A. Niemi and G. Semenoff, Nucl. Phys. B251, 15 (1985); A. Niemi and G. Semenoff, Phys. Rep. 135, 99 (1986).

[18] See, for example, H. Umezawa; Advanced Field Theory: Micro, Macro, and Thermal Physics (AIP, New York, 1993). 